\title{
Cost-effectiveness analysis of the management of distal ureteral stones in children
}

Katherine H. Chan ${ }^{a, b}$, Benjamin M. Whittam ${ }^{a}$, Amy Krambeck ${ }^{a}$, MD, Stephen M. Downs, MD, MS $^{c}$, Rosalia Misseri ${ }^{a}$, Mark P. Cain ${ }^{a}$, William E. Bennett, Jr., MD, MS ${ }^{b, d}$

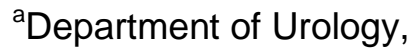

Department of Pediatrics: ${ }^{\mathrm{b}}$ Center for Pediatric and Adolescent Comparative Effectiveness Research, 'Section of Children's Health Services Research, 'Section of Gastroenterology, Hepatology and Nutrition

Indiana University School of Medicine, Indianapolis, Indiana

\author{
Corresponding Author: \\ Katherine H. Chan, MD, MPH
}

a705 Riley Hospital Drive, Riley Outpatient Center, 4230, Indianapolis, IN 46202

Phone: 216-269-7878; Fax:001-317-944-7481; e-mail: kchubert@iupui.edu

Abstract word count: 262; Manuscript word count: 2,340

Key words: cost-benefit analysis; urolithiasis

Objective: To determine the most cost-effective approach to the management of distal ureteral stones in children given the potential for recurrent renal colic during a trial of passage versus potential stent discomfort and complications of ureteroscopy.

Methods: We developed a decision tree to project costs and clinical outcomes associated with observation, medical explusive therapy (MET), and ureteroscopy for the management of an index patient with a $4 \mathrm{~mm}$ distal ureteral stone. We determined which strategy would be least costly and offer the most pain-free days within 30 days of diagnosis. We performed a one-way sensitivity analysis on the probability of successful stone passage with MET. We obtained probabilities from the literature and costs from the 2016 Pediatric Health Information System Database.

Results: Ureteroscopy was the costliest strategy but maximized the number of pain-free days within 30 days of diagnosis ( $\$ 5,282 / 29$ pain-free days). MET was less costly than ureteroscopy but also less effective (\$615/21.8 pain-free days). Observation cost more than MET and was 
also less effective ( $\$ 2,139 / 15.5$ pain-free days). The one-way sensitivity analysis on the probability of successful stone passage with MET demonstrated that ureteroscopy always has the highest net monetary benefits value and is therefore the recommended strategy given a fixed WTP.

Discussion: Using a rigorous decision-science approach, we found that ureteroscopy is the recommended strategy in children with small distal ureteral stones. Although it cost more than MET, it resulted in more pain-free days in the first 30 days following diagnosis given the faster resolution of the stone episode.

\section{Introduction}

The incidence of nephrolithiasis is increasing in children and the economic impact is significant. [1, 2] The management options for children who present with ureteral stones include: (1) a trial of spontaneous passage, (2) medical expulsive therapy (MET), (3) ureteroscopy, and (4) extracorporeal shock wave lithotripsy (SWL). The 2016 American Urological Association guidelines recommend a trial of spontaneous passage or MET for children with uncomplicated ureteral stones less than or equal to $10 \mathrm{~mm}$.[3] Parents may elect ureteroscopy or SWL for children with proximal or mid ureteral stones who cannot pass their stone spontaneously.[3] The guidelines recommend ureteroscopy for children with distal ureteral stone that fail a trial of observation or MET. SWL is not indicated for these patients due to poor penetration of shock waves in the pelvis and limited efficacy. In addition, there are concerns about its long-term adverse effects and evidence of decreasing utilization.[4, 5]

In a recent meta-analysis, pediatric patients who received alpha-blockers as part of MET had 2.2 times the odds of stone passage within 6 weeks compared to those who did not.[6] There is evidence, however, that physicians do not prescribe MET consistently in children.[7] In one retrospective multi-institutional cohort study of pediatric patients with ureteral stones up to $10 \mathrm{~mm}$, only $36 \%$ of them received MET.[8] There are potential disadvantages to observation, MET and ureteroscopy. Patients who elect observation or MET may have recurrent episodes of renal colic resulting in repeated visits to the clinic or Emergency Department (ED). They also may limit activities and/or miss school while awaiting spontaneous stone passage. In a study of adults with ureteral stones, $11 \%$ visited the ED again within 30 days of stone diagnosis.[9] Ureteroscopy is more costly than the other options and patients may experience stent-related discomfort postoperatively.

The objective of our study was to compare the cost-effectiveness of three strategies for the management of ureteral stones in children: (1) observation, (2) MET, and (3) ureteroscopy. Our goal was to determine which strategy would be least costly and offer the most pain-free days within 30 days of diagnosis (i.e. most cost-effective).

\section{Methods}




\section{Model structure}

We developed a decision tree (Figure 1) to project costs and clinical outcomes associated with the management of uncomplicated, small, distal ureteral stones in children from the perspective of the healthcare system. We defined "uncomplicated" patients as those with no signs of sepsis or acute renal failure, no solitary kidney, and no bilateral obstruction. We compared three strategies, (1) observation, (2) MET, and (3) ureteroscopy. We defined MET as a 14-day trial of stone passage with a daily alpha-blocker such as tamsulosin. The model simulated costs and clinical outcomes for a hypothetical 10-year old female patient with a $4 \mathrm{~mm}$ distal ureteral stone (Figure 1). We estimated the total number of pain-free days within the first 30 days following the onset of acute renal colic.

Key assumptions

Some of the key simplifying assumptions in this model included the following:

(1) There were no stones in the kidney.

(2) There was no evidence of urinary tract infection,

(3) If the patient did not spontaneously pass the stone within 14 days of diagnosis (in either MET or observation branches) she would undergo ureteroscopy.

(4) The urologist always achieved retrograde access for ureteroscopy without active or passive dilation. (The model assumes that a variety of ureteroscopes are available including ultra-thin 4.5 Fr semi-rigid ureteroscopes).[10-12]

(5) The urologist always placed a ureteral stent after ureteroscopy.

(6) All patients removed the ureteral stent with a string at home.

(7) One ureteroscopy was required for stone clearance.

(8) All patients were stone-free within 30-days of stone diagnosis.

\section{Model parameterization}

Appendix 1 shows the base case values for selected model parameters. To the extent possible, we used published scientific articles to derive these parameter estimates. Models were created using TreeAge Pro 2017 (TreeAge Software, Inc., Williamstown, MA). We did not seek Institutional Review Board approval for this study because we used only publicly available resources for parameterizing our model.

\section{Probabilities}

We used specific probabilities from the pediatric and adult stone literature to define the likelihood of the index patient progressing to each branch in the decision tree (Appendix 1). We extrapolated probabilities from the adult literature when pediatric data were not available. In the MET and observation branches we modeled the probability of a visit to 
the ED within 30 days of diagnosis of the symptomatic ureteral stone.[9] In the MET branch, we modeled the probability of a MET-related adverse event using data from a systematic review of medical expulsive therapy in children.[6] We assumed that the cost of a MET-related adverse event was roughly equivalent to the cost of a visit to the ED. We determined the probability of spontaneous stone passage and median time to passage in the MET and observation branches from a randomized controlled trial of tamsulosin in children with ureteral stones.[13] We determined the probability of perioperative ureteroscopy-related complications based on the incidence of Satava and Clavien grade 3 complications from a systematic review of pediatric ureteroscopy studies.[14, 15] We assumed that the cost associated with a perioperative complication was roughly equivalent to a 23 -hour hospital stay. Given that surgery might be required, a) the cost of a 23-hour hospital stay alone likely represents the minimal cost of managing a perioperative complication and $b$ ) our assumption biases in favor of ureteroscopy. We also modeled the probability of perioperative anesthetic adverse events requiring rapid response assistance.[16] We assumed that patients who had a perioperative ureteroscopic or anesthetic-related adverse events would be admitted to the hospital for a 23-hour observation. Finally, we modeled the probability of a visit to the ED within 30 days after the ureteroscopy based on data from a large series of adult patients.[17]

\section{Costs}

We derived costs associated with the various health states in the model primarily from the Pediatric Health Information System, an administrative database from approximately 54 U.S. children's hospitals (Appendix 2).[18] We extracted median adjusted Ratio of Cost to Charges (RCC) based costs for common outpatient kidney stone procedures from January 1, 2016 to December 31, 2016. We determined the median cost of outpatient ureteroscopy with lasen lithotripsy and ureteral stent placement at United States children's hospitals in 2016. We excluded patients with concurrent procedures in order to avoid inclusion of non-stone procedures such as pyeloplasty that commonly involve ureteral stent placement. We assumed that costs for adverse events associated with MET were equivalent to one ED visit. We calculated the median cost of a visit to the ED for any reason at U.S. children's hospitals in 2016, as a precise estimate of an ED visit for a medication side effect was difficult to ascertain. We also included costs of ED visits during a trial of spontaneous passage (with or without MET) and the costs of ED visits post-ureteroscopy. We assumed that costs for a perioperative complication were roughly equivalent to a 23 -hour admission to the hospital. We used Access Medicine to determine the cost of a 30-day supply of tamsulosin.[19]

\section{Outcomes assessed}

The primary outcomes assessed were the costs and clinical outcomes among children with symptomatic distal ureteral stones. We estimated the total number of pain-free days within the first 30 days following the onset of acute renal colic. First, we determined the number of symptomatic stone days defined as the time from the 
diagnosis of the symptomatic ureteral stone to the day of stone clearance (Appendix 3). We considered patients "stone-free" after they passed the stone spontaneously or removed the stent following ureteroscopy. We subtracted the number of symptomatic stone days from thirty in order to determine the number of pain-free days within 30 days of diagnosis.

We performed a one-way sensitivity analysis in which we varied the probability of successful stone passage with MET from 0 to 1 . We performed a net benefits calculation, combining cost, effectiveness and willingness-to-pay (WTP) $(\$ 50,000)$ into a single value. The strategy with the highest net benefits value is the recommended strategy given a fixed WTP. If one strategy's line is always the highest on the vertical benefits scale, there are no CE thresholds in that case for the given variable range and WTP.

Figure 1 depicts the initial branches of the decision tree including the probability of a repeated ED visit within the first 30 days after diagnosis of the ureteral stone. There are also branches modeling the probability of an adverse event related to MET and the probability of a perioperative complication at the time of ureteroscopy. In addition, we modeled the probability of a repeated ED visit following ureteroscopy. Finally, if the index patient failed a trial of observation or MET, she progressed to ureteroscopy in the model.

\section{Results}

\section{Base-case results}

Under base-case assumptions, ureteroscopy was the costliest strategy but maximized the number of pain-free days within 30 days of diagnosis ( $\$ 5,282 / 29$ pain-free days) (Table 1). MET was less costly than ureteroscopy but also less effective $(\$ 615 / 21.8$ pain-free days). Observation cost more than MET and was also less effective ( $\$ 2,139 / 15.5$ pain-free days). Therefore, the observation strategy is dominated by MET. Figure 2 depicts the cost-effective frontier which is a line connecting the two strategies, ureteroscopy and MET, that are not dominated. The willingness-to-pay slope intersects with the favored strategy which, in this case, is ureteroscopy.

\section{Sensitivity analysis}

The one-way sensitivity analysis on the probability of successful stone passage with MET demonstrated that ureteroscopy always has the highest net monetary benefits value (i.e, the lines for MET and ureteroscopy do not intersect) and is therefore the recommended strategy given a fixed WTP.

\section{Discussion}

Using a rigorous decision-science approach, we found that ureteroscopy is the recommended strategy in children with small distal ureteral stones. Although it cost more than MET, it resulted in more pain-free days in the first 30 days following 
diagnosis given the faster resolution of the stone episode. We also noted that MET is always more effective and less costly than observation and thus should be utilized in all eligible children. URS maximizes the number of pain-free days within the first 30 days after diagnosis and costs approximately $\$ 4600$ more than MET and approximately $\$ 1500$ more than observation. The increased probability of spontaneous passage and decreased time to stone passage likely explains the decreased cost of MET compared to observation since patients have fewer opportunities for ED visits. The cost of a 30day supply is nominal and MET-related adverse events are rare.

Although ureteroscopy was the preferred strategy in our study for managing small distal ureteral stones in children, it has several disadvantages including stent discomfort, perioperative complications, and increased cost. Tamsulosin is still a reasonable option for pediatric patients with small distal ureteral stones who wish to avoid the risks of surgery and the potential sequelae of ureteral stent placement. Despite evidence of the safety and efficacy of tamsulosin, it is underutilized for children with ureteral stones.[7] A recent study using commercial claims data found that only $15 \%$ of children evaluated for ureteral stones in the ED filled a prescription for MET within one week of their visit.[7] Joshi and colleagues estimated that approximately $80 \%$ of adults experience stent discomfort that has a negative impact on quality of life.[20] Therefore, identification of patients most likely to benefit from MET could help avoid some of the adverse effects associated with ureteroscopy and ureteral stenting.

Meta-analyses of numerous randomized controlled trials of tamsulosin for adults with ureteral stones confirmed that patients treated with tamsulosin have higher stone expulsion rates and lower analgesic requirements.[21-23] Several recent high quality, large randomized controlled trials, however, have questioned the clinical effectiveness of tamsulosin for MET.[24, 25] The Spontaneous Urinary Stone Passage by Drugs (SUSPEND) trial found that neither tamsulosin nor nifedipine decreased the need for treatment of ureteral stones to achieve stone clearance after four weeks.[25] Another multi-center, phase III trial found no benefit of tamsulosin for patients with distal ureteral stones less than or equal to $10 \mathrm{~mm}$ in terms of spontaneous passage, time to stone passage or analgesic requirements.[24] In a subgroup analysis of patients with larger distal ureteral stones, however, tamsuloin increased stone passage.[24] Ye and colleagues recently published the largest multi-center, randomized, double blind trial to date comparing comparing tamsulosin to placebo.[26] Patients in the tamsulosin group had a higher stone expulsion rate, a shorter time to expulsion and lower use of analgesics.[26] Subgroup analysis identified a specific benefit for tamsulosin for the treatment of large distal ureteral stones $(>5 \mathrm{~mm})$. Furthermore, a recent meta-analysis of three randomized controlled trials and two retrospective cohort studies in pediatric patients found that MET increased the odds of stone passage by approximately two-fold compared to observation.[6]

Prior cost-effectiveness studies in the adult stone literature have compared ureterosopy to SWL for renal calculi.[27, 28] In an instrumental variable analysis, 
Hollingsworth and colleagues noted that expenditures were significantly lower after MET versus endoscopic stone removal in patients with ureteral stones.[29] To our knowledge, there are no prior cost-effectiveness analyses in the adult or pediatric stone literature comparing observation, MET and ureteroscopy.

The primary limitation of this study is the relative paucity of reliable data in children to estimate parameters in our model. We made several assumptions about probability and costs which might limit the generalizability of our results to some populations and clinical scenarios. As such, we included data from small, pediatric randomized controlled trials comparing MET to observation and we extrapolated data from the adult literature when pediatric data were not available. Other límitations include the omission of parent and patient-centered outcomes such as missed days of work/school and stent-related discomfort. We also did not use utilities for the decision tree since there are no published utilities for pediatric stone disease. To our knowledge, there are no published utilities for certain stone procedures such as ureteroscopy even in the adult population.

\section{Conclusions}

In children with small distal ureteral stones, ureteroscopy is the preferred strategy and medical expulsive therapy is superior to observation. Future studies are needed to determine parental/child preferences in the management of pediatric stone disease and to expand our knowledge of the costs and probabilities used to parameterize the model.

\section{Figure Legends}

Figure 1: Decision tree with initial branches

Figure 2: Cost-effectiveness analysis of options for the management of small distal ureteral stones in children

Figure 3: One-way sensitivity analysis on the probability of stone passage with medical expulsive therapy

\section{Conflict of Interest}

None

\section{Funding source}

None

\section{Ethical approval}

Not required 


\section{REFERENCES}

[1] Wang HH, Wiener JS, Lipkin ME, Scales CD, Jr., Ross SS, Routh JC. Estimating the nationwide, hospital based economic impact of pediatric urolithiasis. The Journal of urology. 2015;193:1855-9.

[2] Tasian GE, Ross ME, Song L, Sas DJ, Keren R, Denburg MR, et al. Annual Incidence of Nephrolithiasis among Children and Adults in South Carolina from 1997 to 2012. Clin J Am Soc Nephrol. 2016;11:488-96. [3] Assimos D, Krambeck A, Miller NL, Monga M, Murad MH, Nelson CP, et al. Surgical Management of Stones: American Urological Association/Endourological Society Guideline, PART II. The Journal of urology. 2016;196:1161-9.

[4] Denburg MR, Jemielita TO, Tasian GE, Haynes K, Mucksavage P, Shults J, et al. Assessing the risk of incident hypertension and chronic kidney disease after exposure to shock wave lithotripsy and ureteroscopy. Kidney Int. 2016;89:185-92.

[5] Wang HH, Huang L, Routh JC, Nelson CP. Shock wave lithotripsy vs ureteroscopy: variation in surgical management of kidney stones at freestanding children's hospitals. The Journal of urology.

2012;187:1402-7.

[6] Velazquez N, Zapata D, Wang HH, Wiener JS, Lipkin ME, Routh JC. Medical expulsive therapy for pediatric urolithiasis: Systematic review and meta-analysis. J Pediatr Urol. 2015;11:321-7.

[7] Ellison JS, Merguerian PA, Fu BC, Holt SK, Lendvay TS, Gore JL, et al. Use of medical expulsive therapy in children: An assessment of nationwide practice patterns and outcomes. J Pediatr Urol. 2017;13:509 e1- e7.

[8] Tasian GE, Cost NG, Granberg CF, Pulido JE, Rivera M, Schwen Z, et al. Tamsulosin and spontaneous passage of ureteral stones in children: a multi-institutional cohort study. The Journal of urology. 2014;192:506-11.

[9] Ganesan V, Loftus CJ, Hinck B, Greene DJ, Nyame YA, Sivalingam S, et al. Clinical Predictors of 30-Day Emergency Department Revisits for Patients with Ureteral Stones. The Journal of urology. 2016;196:1467-70.

[10] Atar M, Sancaktutar AA, Penbegul N, Soylemez H, Bodakci MN, Hatipoglu NK, et al. Comparison of a 4.5 F semi-rigid ureteroscope with a 7.5 F rigid ureteroscope in the treatment of ureteral stones in preschool-age children. Urological research.2012;40:733-8.

[11] Kocaoglu C, Ozkan KU. The effectiveness of $4.5 \mathrm{~F}$ ultrathin semirigid ureteroscope in the management of ureteral stones in prepubertal children: is there a need for any ureteral dilatation? Urology. 2014;84:202-5.

[12] Kucukdurmaz F, Efe E, Sahinkanat T, Amasyali AS, Resim S. Ureteroscopy With Holmium:Yag Laser Lithotripsy for Ureteral Stones in Preschool Children: Analysis of the Factors Affecting the Complications and Success. Urology. 2018;111:162-7.

[13] Mokhless 1, Zahran AR, Youssif M, Fahmy A. Tamsulosin for the management of distal ureteral stones in children: a prospective randomized study. J Pediatr Urol. 2012;8:544-8.

[14] Ishii H, Griffin S, Somani BK. Flexible ureteroscopy and lasertripsy (FURSL) for paediatric renal calculi: results from a systematic review. J Pediatr Urol. 2014;10:1020-5.

[15] Dogan HS, Onal B, Satar N, Aygun C, Piskin M, Tanriverdi O, et al. Factors affecting complication rates of ureteroscopic lithotripsy in children: results of multi-institutional retrospective analysis by Pediatric Stone Disease Study Group of Turkish Pediatric Urology Society. The Journal of urology. 2011;186:1035-40.

[16] Schleelein LE, Vincent AM, Jawad AF, Pruitt EY, Kreher GD, Rehman MA, et al. Pediatric perioperative adverse events requiring rapid response: a retrospective case-control study. Paediatr Anaesth. 2016;26:734-41.

[17] Tang L, Gao X, Xu B, Hou J, Zhang Z, Xu C, et al. Placement of ureteral stent after uncomplicated ureteroscopy: do we really need it? Urology. 2011;78:1248-56. 
[18] PHIS. Children's Hospital Association; 2018.

[19] Access Medicine. McGraw-Hill Global Education.

[20] Joshi HB, Okeke A, Newns N, Keeley FX, Jr., Timoney AG. Characterization of urinary symptoms in patients with ureteral stents. Urology. 2002;59:511-6.

[21] Hollingsworth JM, Canales BK, Rogers MA, Sukumar S, Yan P, Kuntz GM, et al. Alpha blockers for treatment of ureteric stones: systematic review and meta-analysis. BMJ. 2016;355:i6112.

[22] Hollingsworth JM, Rogers MA, Kaufman SR, Bradford TJ, Saint S, Wei JT, et al. Medical therapy to facilitate urinary stone passage: a meta-analysis. Lancet. 2006;368:1171-9.

[23] Campschroer T, Zhu Y, Duijvesz D, Grobbee DE, Lock MT. Alpha-blockers as medical expulsive therapy for ureteral stones. Cochrane Database Syst Rev. 2014:CD008509.

[24] Furyk JS, Chu K, Banks C, Greenslade J, Keijzers G, Thom O, et al. Distal Ureteric Stones and Tamsulosin: A Double-Blind, Placebo-Controlled, Randomized, Multicenter Trial. Ann Emerg Med. 2016;67:86-95 e2.

[25] Pickard R, Starr K, MacLennan G, Lam T, Thomas R, Burr J, et al. Medical expulsive therapy in adults with ureteric colic: a multicentre, randomised, placebo-controlled trial. Lancet. 2015;386:341-9.

[26] Ye Z, Zeng G, Yang H, Tang K, Zhang X, Li H, et al. Efficacy and Safety of Tamsulosin in Medical Expulsive Therapy for Distal Ureteral Stones with Renal Colic: A Multicenter, Randomized, Double-blind, Placebo-controlled Trial. Eur Urol. 2017.

[27] Koo V, Young M, Thompson T, Duggan B. Cost-effectiveness and efficiency of shockwave lithotripsy vs flexible ureteroscopic holmium:yttrium-aluminium-garnet laser lithotripsy in the treatment of lower pole renal calculi. BJU Int. 2011;108:1913-6.

[28] Cone EB, Eisner BH, Ursiny M, Pareek G. Cost-effectiveness comparison of renal calculi treated with ureteroscopic laser lithotripsy versus shockwave lithotripsy. JEndourol. 2014;28:639-43.

[29] Hollingsworth JM, Norton EC, Kaufman SR, Smith RM, Wolf JS, Jr., Hollenbeck BK. Medical expulsive therapy versus early endoscopic stone removal for acute renal colic: an instrumental variable analysis.

The Journal of urology. 2013;190:882-7. 

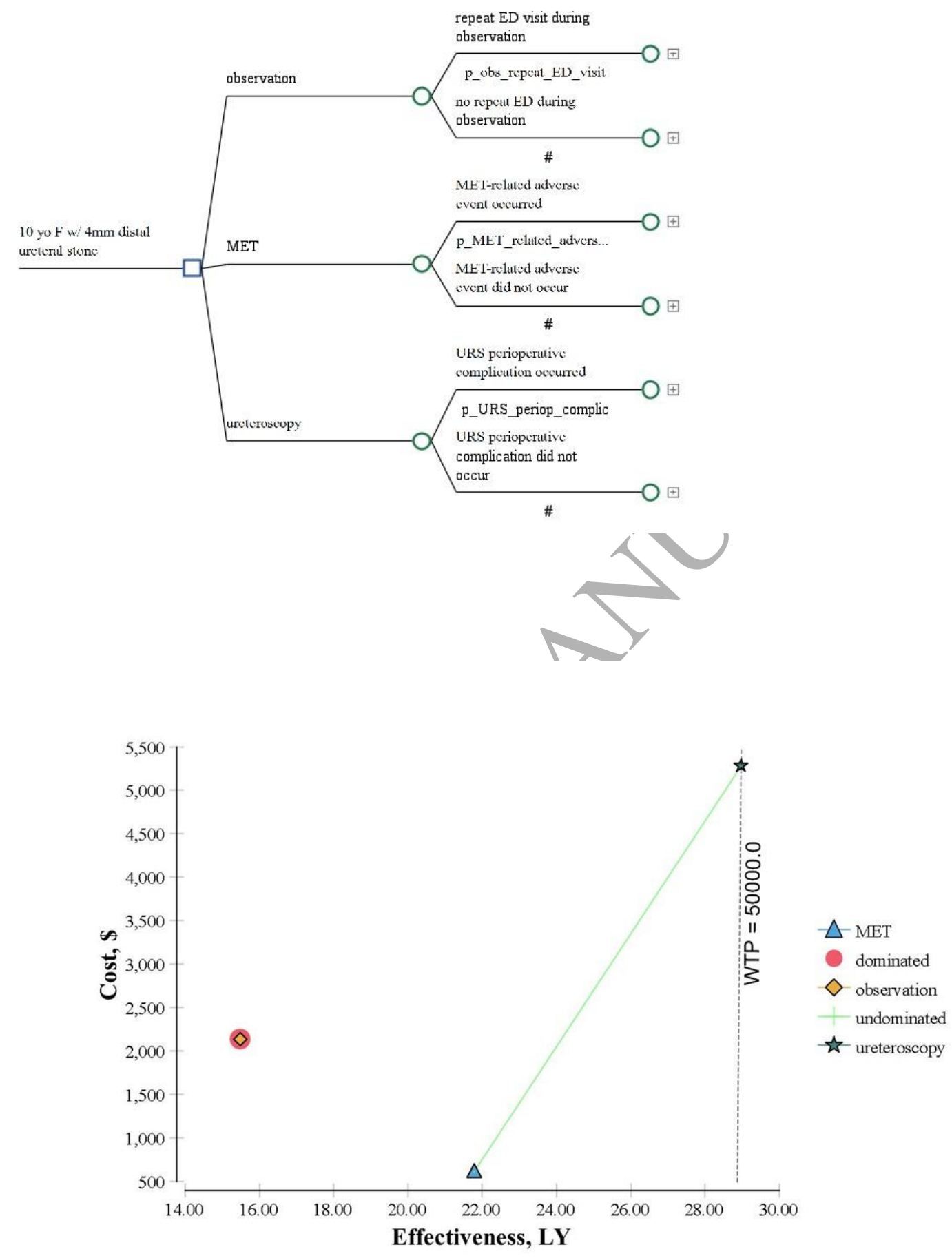


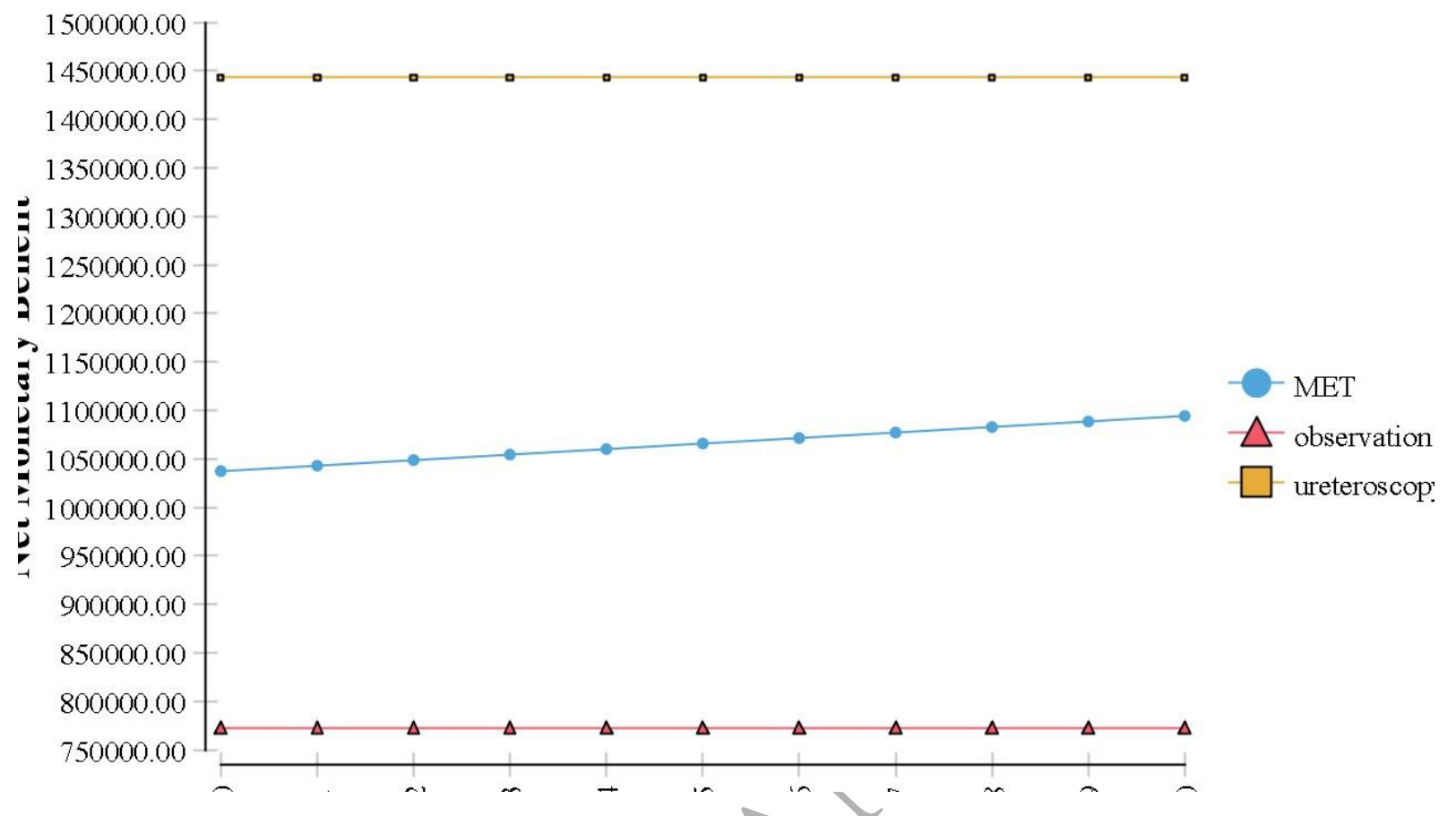

Table 1: Cost-effectiveness of three strategies for management of small, distal ureteral stones in children

\begin{tabular}{|l|l|c|l|l|l|l|}
\hline & \multicolumn{1}{|c|}{ Cost } & $\begin{array}{c}\text { Incr. } \\
\text { cost }\end{array}$ & $\begin{array}{c}\text { Ave. } \\
\text { effectiveness }\end{array}$ & $\begin{array}{c}\text { Incr. } \\
\text { effectiveness }\end{array}$ & $\begin{array}{c}\text { Ave. cost- } \\
\text { effectiveness }\end{array}$ & \multicolumn{1}{|c|}{ ICER } \\
\hline${ }^{*}$ MET & $\$ 615$ & & 21.8 & & 28.2 & 0 \\
\hline${ }^{* \star}$ OBS & $\$ 2,139$ & $\$ 1,524$ & 15.5 & -6.30 & 138.2 & -241.8 \\
\hline${ }^{\star \star \star}$ URS & $\$ 5,282$ & $\$ 4,667$ & 29.0 & 7.19 & 182.3 & 649.4 \\
\hline
\end{tabular}

${ }^{\star}$ MET=medical expulsive therapy

${ }^{\star \star}$ OBS = observation

${ }^{\star \star}$ URS=ureteroscopy

Ave. $=$ Average

Incr. = Incremental 